\title{
A Blueberry Extract Supplemented Diet Partially Restores $\alpha$-Synuclein-Dependent Lifespan Loss and Developmental Defects in Drosophila
}

\author{
David B. Lipsett, Brian E. Staveley* \\ Department of Biology, Memorial University of Newfoundland, St. John's, Canada \\ Email: ${ }^{*}$ bestave@mun.ca
}

Received 15 March 2014; revised 17 April 2014; accepted 25 May 2014

Copyright (C) 2014 by authors and Scientific Research Publishing Inc.

This work is licensed under the Creative Commons Attribution International License (CC BY).

http://creativecommons.org/licenses/by/4.0/

(c) (i) Open Access

\begin{abstract}
Oxidative stress has been strongly associated with Parkinson disease (PD) aetiology. We investigated the effects of blueberry extract (BBE) supplementation on $\alpha$-synuclein induced phenotypes in a Drosophila melanogaster model of PD. Enhanced $\alpha$-synuclein expression in D. melanogaster dopaminergic (DA) neurons can reduce lifespan and we have performed longevity assays to measure the effects of BBE on $D$. melanogaster survival. Flies expressing $\alpha$-synuclein in their DA neurons fed BBE had up to an 8 day, or $15 \%$, greater median lifespan than those fed a standard control diet. In addition, BBE improved $\alpha$-synuclein-induced developmental defects in the Drosophila eye. Our biometric analyses revealed that individuals fed BBE had less atypical ommatidia as well as an increased number of mechanosensory bristle cells than those fed a control diet. We propose that BBE, rich in naturally occurring antioxidants, promotes the survival of neurons in tissues with increased levels of $\alpha$-synuclein through a protective cell survival mechanism.
\end{abstract}

\section{Keywords}

Drosophila melanogaster, Blueberry Extract, $\alpha$-Synuclein, Model of Parkinson Disease, Lifespan, Ommatidia Development

\section{Introduction}

Parkinson disease (PD) is the second most common progressive neurodegenerative disorder and is only surpassed in frequency by Alzheimer disease [1]. The pathophysiological hallmarks of PD include the loss of do-

${ }^{*}$ Corresponding author.

How to cite this paper: Lipsett, D.B. and Staveley, B.E. (2014) A Blueberry Extract Supplemented Diet Partially Restores $\alpha$-Synuclein-Dependent Lifespan Loss and Developmental Defects in Drosophila. Advances in Parkinson's Disease, 3, 3-9. 
paminergic (DA) neurons in the substantia nigra pars compacta $(S N c)$ and the presence of intra-neuronal inclusions known as Lewy bodies (LB) in surviving cells. Affected individuals have both motor and non-motor symptoms ranging from bradykinesia, resting tremor, and muscular rigidity to dementia, depression and olfactory dysfunction. Initially believed to be an entirely sporadic disease, linkage studies identified $\alpha$-synuclein (PARK 1 and 4) as the first gene related to PD [2]. The human $\alpha$-synuclein gene (SNCA) encodes a 140 amino acid peripheral membrane protein that localizes to the pre-synaptic region of neurons [3]. Both point mutations and duplications of its gene locus result in autosomal-dominant PD, the latter causing a more severe early-onset form of the disease. Additionally, both LBs and Lewy neurites, located in the perikarya and neuronal processes, respectively, stain positively for $\alpha$-synuclein. $\alpha$-synuclein is associated with both sporadic and familial PD and seems to play a critical role in its origin.

Oxidative stress is consistently associated with the pathogenesis of PD; however, its role in disease progression remains unclear. A cell undergoes oxidative stress when the net balance between the generated reactive oxygen species (ROS) and the available antioxidant defense mechanisms favour the former. Post-mortem analysis of PD patient brains reveals higher levels of oxidative stress biomarkers like dysfunctional mitochondria, decreased levels of reduced glutathione, and deficiencies in antioxidant enzymes in the $S N$ of affected individuals [4]. Recent evidence suggests pluripotent stem cell-derived DA neurons from a PD patient with a SNCA triplication accumulate $\alpha$-synuclein and are susceptible to oxidative stress [5]. This data suggest that the combination of oxidative stress and excess $\alpha$-synuclein may play a pivotal role in the progression of PD.

The toxicity of excess $\alpha$-synuclein appears to be enhanced under conditions of oxidative stress. Studies in Drosophila melanogaster have been especially helpful in elucidating this relationship. Neural expression of $\alpha$-synuclein in Drosophila brains, especially DA neurons, recapitulates the locomotor dysfunctions, age-dependent degeneration of DA neurons, and formation of LBs characteristic of human PD [6]. Decreased lifespan and retinal degeneration have been observed in Drosophila with increased neuronal levels of $\alpha$-synuclein [7]. Co-expression of methionine sulfoxide reductase A (MSRA) or PTEN-induced putative kinase 1 (PINK1), proteins involved in ROS neutralization and damaged mitochondrion turnover, respectively, with $\alpha$-synuclein improves PD-related phenotypes [8]. Given their versatility, Drosophila can help unravel the role of oxidative stress in PD and unveil potential antioxidant therapies.

Blueberries are an excellent source of dietary antioxidants. The therapeutic potential of blueberries in cancer and vascular disease has been described and recent studies in Drosophila suggest that plant extracts may be beneficial to individuals suffering from neurodegenerative diseases [9] [10]. In this study we describe the restorative effects of blueberry extract (BBE) concentrate on a Drosophila model of PD. Enhanced mortality and eye degeneration caused by directed expression of $\alpha$-synuclein in the DA neurons and developing eye, respectively, is improved by supplementing growth media with BBE.

\section{Materials and Methods}

\subsection{Fly Stocks and Culture}

The UAS- $\alpha$-synuclein [6] and Ddc-Gal4 [11] flies were generously provided by Dr. M. Feany (Harvard Medical School) and Dr. J. Hirsh (University of Virginia), respectively. GMR-Gal4 ${ }^{12}$ [12] and UAS-lacZ ${ }^{4-1-2}$ [13] flies were obtained from the Bloomington Drosophila Stock Center at Indiana University. Directed expression of the transgenes in DA neurons and during early eye development was accomplished by crossing homozygous Ddc-Gal4 and GMR-Gal4 females, respectively, to homozygous UAS- $\alpha$-synuclein (PD model) and UAS-lacZ (control) males as per standard methods. To be clear, the UAS-lacZ gene is widely used as a benign responder transgene to control for the consequences of the transcriptional activity of Gal4 and allows for detailed comparison of similarly controlled genes. Flies were fed either a standard cornmeal-yeast-molasses-agar medium (65 g/L cornmeal, $15 \mathrm{~g} / \mathrm{L}$ nutritional yeast extract, $5.5 \mathrm{~g} / \mathrm{L}$ agar, $50 \mathrm{ml} / \mathrm{L}$ fancy grade molasses in water supplemented with $0.1 \mathrm{~g} / \mathrm{ml}$ methyl paraben in ethanol and $2.5 \mathrm{ml}$ propionic acid per L of medium) or standard medium supplemented with either $1 \mathrm{mg} / \mathrm{ml}$ or $5 \mathrm{mg} / \mathrm{ml}$ Webber Naturals' 36:1 concentrate BBE (WN Pharmaceuticals ${ }^{\circledR}$ Ltd., Coquitlam, B.C., V3K 7B5, www.webbernaturals.com).

\subsection{Longevity Assay}

Flies were reared on either standard or BBE supplemented medium (1 or $5 \mathrm{mg} / \mathrm{ml}$ ) then collected under gaseous $\mathrm{CO}_{2}$ every 24 hours until a minimum of 200 adult males of each genotype were obtained. Selected flies were 
then transferred to upright standard plastic shell vials containing the same food medium they were initially exposed to. Each group was maintained at $25^{\circ} \mathrm{C}$ and kept in non-crowded conditions (1 to 20 individuals per vial). Flies were scored for viability every 2 days and transferred to fresh medium without anaesthesia according to established protocol [14]. Survival fractions were calculated in Prism version 5.0b for Mac OS X (GraphPad Software, San Diego California USA, www.graphpad.com) using the product limit (Kaplan-Meier) method.

\subsection{Scanning Electron Microscopy and Biometric Analyses}

Flies were reared and aged 3 to 5 days post-eclosion on either standard or BBE supplemented medium at $29^{\circ} \mathrm{C}$. Surviving flies were preserved at $-80^{\circ} \mathrm{C}$ before being mounted on metal studs under a dissecting microscope. Prepared flies were desiccated overnight and gold coated prior to photography at 170 times magnification with a Hitachi S-570 scanning electron microscope as per standard methods.

All biometric analyses were measured using ImageJ64 version 1.42q [15]. The area of a single ommatidium was determined by dividing the average area of a floret of ommatidia by 7 (data not shown). These numbers were used to distinguish between normal and atypical ommatidia when measuring percent disruption. A disrupted or atypical ommatidium was defined as having an area 50\% smaller or $150 \%$ larger than a typical ommatidium for that condition. An oval with an area between $35000-40000 \mu \mathrm{m}^{2}$ was overlaid on the flattest portion of each analyzed eye with Paintbrush version 2.1.1 for Mac OS X (Copyright @ 2007-2010 Soggy Waffles). Individual disrupted areas within the oval were measured in triplicate and a percent value was obtained by dividing the summed average values into the average area of the oval (measured in triplicate). $n=15$ for each analyzed condition for both bristle counts and ommatidium area measurements, whereas $n=10$ for the percent disruption analysis. Bar graphs were produced using Prism version 5.0b for Mac OS X (GraphPad Software, San Diego California USA, www.graphpad.com).

\section{Results}

\subsection{Increased Concentrations of Blueberry Extract Protect against $\alpha$-Synuclein-Induced Early Mortality}

Here we report a reduced lifespan in flies when $\alpha$-synuclein expression is enhanced in the DA neurons (Figure 1 (a)). The median survival time of $\alpha$-synuclein-expressing flies was reduced by $37 \%$ compared to the lac $Z$ cohort when both groups were fed a control diet. A diet rich in BBE partially rescued the reduced lifespan caused by increased neuronal amounts of $\alpha$-synuclein in D. melanogaster (Figure 1(b)). $\alpha$-synuclein-expressing flies fed a diet containing $5 \mathrm{mg} / \mathrm{ml} \mathrm{BBE}$ had an 8-day (15\%) greater median lifespan than those fed a control diet. A similar result was not found with a concentration of $1 \mathrm{mg} / \mathrm{ml} \mathrm{BBE}$. The median survival values for each group are found in Table 1.

\subsection{Blueberry Extract Suppresses $\alpha$-Synuclein-Induced Developmental Defects in the Drosophila Eye}

A rough external eye phenotype occurs when GMR-Gal4 is used to drive expression of $\alpha$-synuclein in the developing Drosophila eye (Figure 2(b)) [8]. BBE supplementation restores mean $\alpha$-synuclein-induced defects to control levels (Figure 2(e)). The mean disruption of lacZ-expressing flies fed a control diet was $29 \%$ of the analyzed area. In $\alpha$-synuclein-expressing flies, the mean disruption was reduced from $73 \%$ for those fed a con-

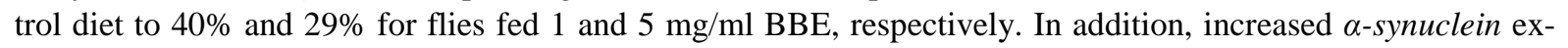
pression reduced the mean number of neurosensory bristles per eye to 373 in flies fed a control diet, whereas the eyes of lacZ-expressing flies on the same diet contained 541 (Figure 2(e)). The mean number of bristles per eye was raised to 415 and 438 when the $\alpha$-synuclein-expressing flies were raised on a diet consisting of 1 and 5 $\mathrm{mg} / \mathrm{ml} \mathrm{BBE}$, respectively. This provides another example of BBE-induced protection in a Drosophila tissue that is rich in neurons.

\section{Discussion}

Recent evidence has suggested that a diet rich in blueberries may help slow the age-related degeneration of neurons. In a human study, blueberry juice supplementation improved memory function in older adults with early 


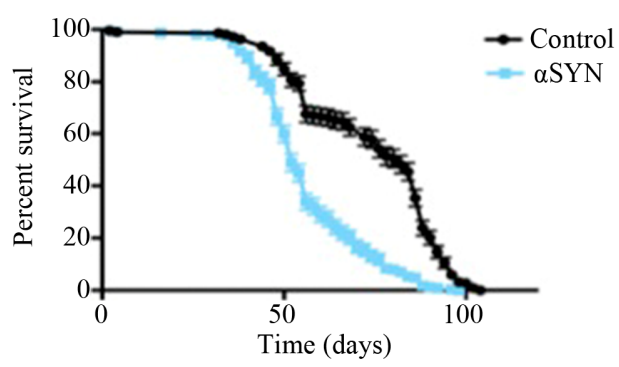

(a)

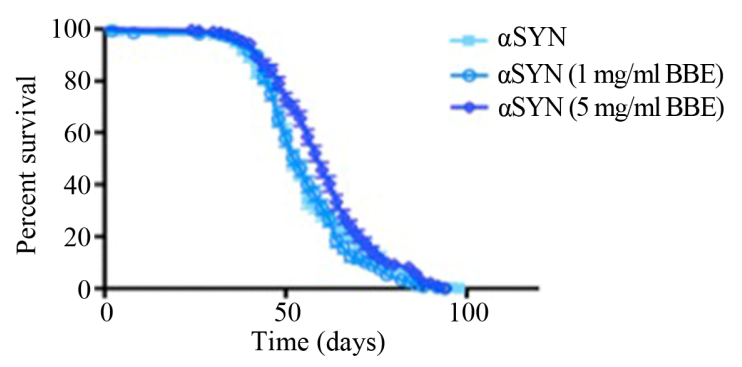

(b)

Figure 1. Blueberry extract partially protects Drosophila melanogaster against $\alpha$-synuclein-induced early mortality. Directed expression of $\alpha$-synuclein $(\mathrm{n}=218)$ in DA neurons shortens lifespan in Drosophila fed a standard diet, as compared to a lacZ $(\mathrm{n}=227)$ control $(\mathrm{p}<0.05)$. Flies fed diets containing $5 \mathrm{mg} / \mathrm{ml} \mathrm{BBE}(\mathrm{n}=$ 283) were partially protected against the $\alpha$-synuclein-induced mortality $(\mathrm{p}<0.05)$, whereas $1 \mathrm{mg} / \mathrm{ml} \mathrm{BBE}(\mathrm{n}=$ 267 ) had no significant effect. Genotypes are $w^{1118}$; UAS-lacZ/Ddc-Gal4 (control) and $w^{1118}$; UAS- $\alpha$-synuclein/Ddc-Gal4 $(\alpha \mathrm{SYN})$. Errors bars represent standard error of the mean. p-values were calculated by the log-rank (Mantel-Cox) test and multiple comparisons were corrected for using the Bonferroni method.

Table 1. Median survival times of transgenic Drosophila melanogaster reared on either a standard or blueberry extract-supplemented diet.

\begin{tabular}{cc}
\hline Genotype (food medium) & Median survival (days) \\
\hline$w^{1118} ;$ UAS-lacZ/Ddc-Gal4 (control) & $82^{\mathrm{a}}$ \\
$w^{1118} ;$ UAS- $\alpha$-syn/Ddc-Gal4 (control) & $52^{\mathrm{b}}$ \\
$w^{1118} ;$ UAS- $\alpha$-syn/Ddc-Gal4 (1 mg/ml BBE) & $54^{\mathrm{b}}$ \\
$w^{1118} ;$ UAS- $\alpha$-syn/Ddc-Gal4 (5 mg/ml BBE) & $60^{\mathrm{c}}$ \\
\hline
\end{tabular}

Groups with different superscripted letters are significantly different from each other $(\mathrm{p}<0.05)$.

memory decline [16]. Furthermore, it has been demonstrated that short-term blueberry supplementation increased heat shock protein 70 (HSP70)-mediated protection against inflammation in aged rat hippocampal cells [17]. These studies suggest that the neuroprotective effects of blueberries or BBE are not confined to D. melanogaster and may extend to mammals.

The Free Radical/Oxidative Stress theory of ageing originated in the 1950's and suggests that an organism ages due, in part, to the accumulation of free radical-induced damage to cellular macromolecules. Previous studies have shown that BBE is capable of ex tending lifespan in D. melanogaster [18]. Our results are novel as we have shown that BBE supplementation can extend lifespan in a Drosophila model of a neurodegenerative disease. The additional antioxidants provided by BBE supplementation may alleviate some of the excess ROS generated during the progression of PD-like cell death resulting in less cellular damage and a longer median survival time in affected flies.

The Drosophila compound eye consists of multiple subunits, or ommatidia, composed of several neurons and peripheral mechanosensory bristle cells. Directed expression of $\alpha$-synuclein during early eye development results in premature degeneration of the retina and abnormal development of external eye morphology [8] [19] Our results suggest that a diet containing BBE protects neurons in the eye against $\alpha$-synuclein-dependent developmental defects. Both the amount of atypical ommatidia and total number of bristles were improved in flies fed a BBE-supplemented diet. A link exists between BBE supplementation and protein turnover via the ubiquitin proteasome system (UPS) as flies fed BBE have increased levels of regulatory particle non-ATPase 11 (Rpn11) mRNA, an essential lid component of the 26S proteasome structure [18]. In humans, the parkin gene (PARK2) encodes an E3 ubiquitin ligase and expression of parkin in Drosophila eyes suppresses $\alpha$-synucleininduced retinal degeneration in older flies [19]. Protein aggregation/turnover is a point of major interest in PD aetiology and the neuroprotective effects of BBE on $\alpha$-synuclein-induced damage in the Drosophila eye may be due in part to increased activity of the UPS system. 

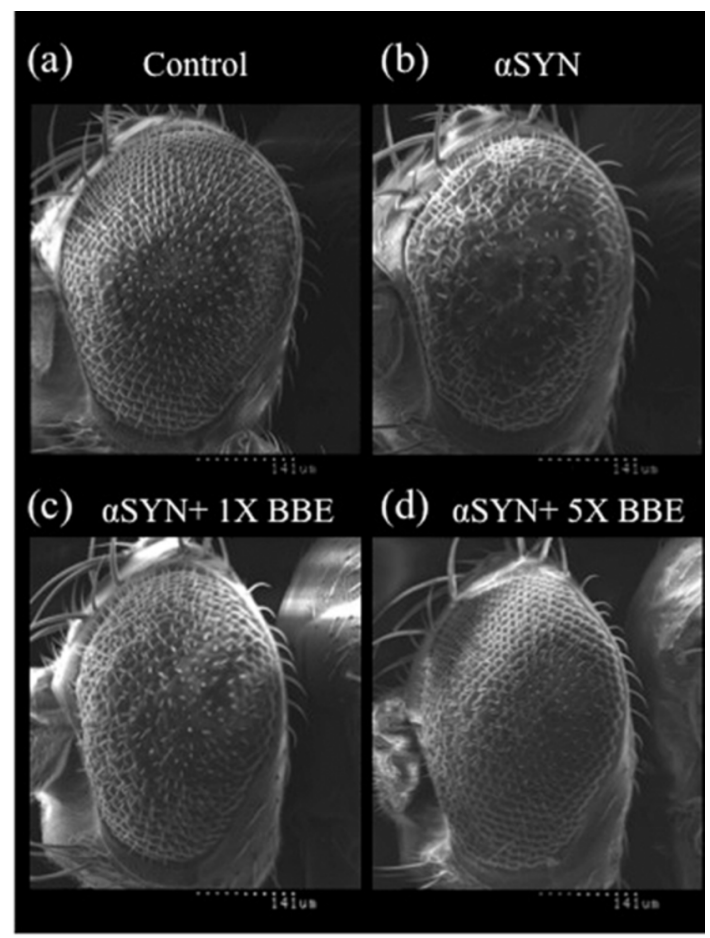

(e)
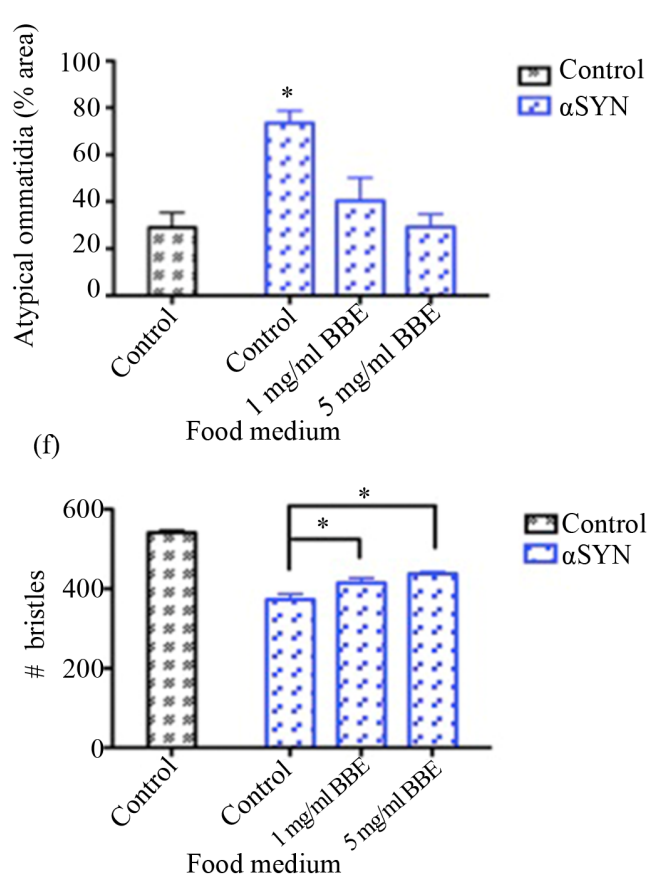

Figure 2. Blueberry extract supplementation counteracts $\alpha$-synuclein-induced developmental defects of the eye. Overexpression of $\alpha$-synuclein during early eye development produces a rough external eye morphology. Flies supplemented with BBE have disruption levels comparable to a lacZ control. Additionally, BBE supplementation partially rescues an $\alpha$-synuclein-induced decrease in mean bristle number. X denotes $\mathrm{mg} / \mathrm{ml}$. *represents $\mathrm{p}<0.05$. Genotypes are $w^{1118}$; UAS-lacZ/GMR-Gal4 (control) and $w^{1118}$; UAS- $\alpha$-synuclein/ GMR-Gal4 ( $\alpha \mathrm{SYN})$. Error bars represent standard error of the mean. p-values were calculated via one-way ANOVA followed by Tukey's Multiple Comparison test.

Our findings may be attributed to a strengthened overall antioxidant defense mechanism in $\alpha$-synuclein-expressing flies. BBE extract can increase the expression of intrinsic antioxidant defense enzymes in $D$. melanogaster that include catalase (Cat), Cu-Zn superoxide dismutase (Sod1), and Mn superoxide dismutase (Sod2) [18]. Additionally, BBE extends lifespan and partially protects wild-type flies under conditions of increased oxidative stress, whereas no effect was reported for Cat or Sod loss-of-function mutants. Similar results have been documented for several other foods high in dietary antioxidants, including extracts of apple polyphenols [20], green tea catechins [21] and black tea [22]. Although dietary antioxidants likely provide an invaluable secondary support to cells undergoing oxidative stress, it appears their protective effects are dependent on intrinsic enzyme antioxidant defense systems.

\section{Conclusion}

Here we present the first demonstration of the neuroprotective effects of BBE in a Drosophila model of $\alpha$-synucleinpathic disease. Previous findings have demonstrated that grape extract improved both the early mortality and premature decline in locomotion in a similar Drosophila model of PD [9]. Taken together, these results epitomize the value of using Drosophila to study PD aetiology. Though the literature is relatively new, studies in this versatile organism have helped develop interest in the potential neuroprotective effects of dietary antioxidants in medical research. Future studies could aim towards unraveling the interaction of dietary antioxidants and the activity of cellular mechanisms, such as the UPS and enzymatic antioxidant pathways.

\section{Acknowledgements}

This research was funded by a Natural Sciences and Engineering Research Council of Canada (NSERC) Canada Graduate Scholarship (NSERC CGS-M) to DBL and an NSERC Discovery Grant to BES. Funding was pro- 
vided by the School of Graduate Studies at Memorial University of Newfoundland to DBL. We thank Dr. Liqui Men (Bio-molecular Imaging Cluster, Memorial University of Newfoundland) for technical assistance with scanning electron microscopy.

\section{References}

[1] de Moura, M.B., dos Santos, L.S. and Van Houten, B. (2010) Mitochondrial Dysfunction in Neurodegenerative Diseases and Cancer. Environmental and Molecular Mutagenesis, 51, 391-405.

[2] Polymeropoulos, M.H., Lavedan, C., Leroy, E., Ide, S.E., Dehejia, A., Dutra, A., et al. (1997) Mutation in the Alpha-Synuclein Gene Identified in Families with Parkinson's Disease. Science, 276, 2045-2047. http://dx.doi.org/10.1126/science.276.5321.2045

[3] Stefanis, L. (2012) Alpha-Synuclein in Parkinson's Disease. Cold Spring Harbor Perspectives in Medicine, 2, Article ID: a009399. http://dx.doi.org/10.1101/cshperspect.a009399

[4] Schapira, A.H. and Jenner, P. (2011) Etiology and Pathogenesis of Parkinson’s Disease. Movement Disorders, 26, 1049-1055. http://dx.doi.org/10.1002/mds.23732

[5] Byers, B., Cord, B., Nguyen, H.N., Schule, B., Fenno, L., Lee, P.C., et al. (2011) SNCA Triplication Parkinson’s Patient's iPSC-Derived DA Neurons Accumulate $\alpha$-Synuclein and Are Susceptible to Oxidative Stress. PLoS One, 6, Article ID: e26159. http://dx.doi.org/10.1371/journal.pone.0026159

[6] Feany, M.B. and Bender, W.W. (2000) A Drosophila Model of Parkinson’s Disease. Nature, 404, 394-398. http://dx.doi.org/10.1038/35006074

[7] Wassef, R., Haenold, R., Hansel, A., Brot, N., Heinemann, S.H. and Hoshi, T. (2007) Methionine Sulfoxide Reductase A and a Dietary Supplement S-Methyl-L-Cysteine Prevent Parkinson's-Like Symptoms. The Journal of Neuroscience, 27, 12808-12816. http://dx.doi.org/10.1523/JNEUROSCI.0322-07.2007

[8] Todd, A.M. and Staveley, B.E. (2008) Pink1 Suppresses Alpha-Synuclein-Induced Phenotypes in a Drosophila Model of Parkinson's Disease. Genome, 51, 1040-1046. http://dx.doi.org/10.1139/G08-085

[9] Long, J.G., Gao, H.X., Sun, L.J., Liu, J.K. and Zhao-Wilson, X. (2009) Grape Extract Protects Mitochondria from Oxidative Damage and Improves Locomotor Dysfunction and Extends Lifespan in a Drosophila Parkinson's Disease Model. Rejuvenation Research, 12, 321-331. http://dx.doi.org/10.1089/rej.2009.0877

[10] Neto, C.C. (2007) Cranberry and Blueberry: Evidence for Protective Effects against Cancer and Vascular Diseases. Molecular Nutrition \& Food Research, 51, 652-664. http://dx.doi.org/10.1002/mnfr.200600279

[11] Li, H., Chaney, S., Forte, M. and Hirsh, J. (2000) Ectopic G-Protein Expression in Dopamine and Serotonin Neurons Blocks Cocaine Sensitization in Drosophila melanogaster. Current Biology, 10, 211-214. http://dx.doi.org/10.1016/S0960-9822(00)00340-7

[12] Freeman, M. (1996) Reiterative Use of the EGF Receptor Triggers Differentiation of all Cell Types in the Drosophila Eye. Cell, 87, 651-660. http://dx.doi.org/10.1016/S0092-8674(00)81385-9

[13] Brand, A.H. and Perrimon, N. (1993) Targeted Gene Expression as a Means of Altering Cell Fates and Generating Dominant Phenotypes. Development, 118, 401-415.

[14] Staveley, B.E., Phillips, J.P. and Hilliker, A.J. (1990) Phenotypic Consequences of Copper-Zinc Superoxide-Dismutase Overexpression in Drosophila melanogaster. Genome, 33, 867-872. http://dx.doi.org/10.1139/g90-130

[15] Abramoff, M.D., Magalhaes, P.J. and Ram, S.J. (2004) Image Processing with ImageJ. Biophotonics International, 11, 36-42.

[16] Krikorian, R., Shidler, M.D., Nash, T.A., Kalt, W., Vinqvist-Tymchuk, M.R., Shukitt-Hale, B., et al. (2010.) Blueberry Supplementation Improves Memory in Older Adults. Journal of Agricultural and Food Chemistry, 58, 3996-4000. http://dx.doi.org/10.1021/jf9029332

[17] Galli, R.L., Bielinski, D.F., Szprengiel, A., Shukitt-Hale, B. and Joseph, J.A. (2006) Blueberry Supplemented Diet Reverses age-Related Decline in Hippocampal HSP70 Neuroprotection. Neurobiol Aging, 27, 344-350. http://dx.doi.org/10.1016/j.neurobiolaging.2005.01.017

[18] Peng, C., Zuo, Y., Kwan, K.M., Liang, Y., Ma, K.Y., Chan, H.Y.E., et al. (2012) Blueberry Extract Prolongs Lifespan of Drosophila melanogaster. Experimental Gerontology, 47, 170-178. http://dx.doi.org/10.1016/j.exger.2011.12.001

[19] Haywood, A.F.M. and Staveley, B.E. (2004) Parkin Counteracts Symptoms in a Drosophila Model of Parkinson's Disease. BMC Neuroscience, 5, 14. http://dx.doi.org/10.1186/1471-2202-5-14

[20] Peng, C., Chan, H.Y., Huang, Y., Yu, H. and Chen, Z.Y. (2011) Apple Polyphenols Extend the Mean Lifespan of Drosophila melanogaster. Journal of Agricultural and Food Chemistry, 59, 2097-2106. http://dx.doi.org/10.1021/jf1046267 
[21] Li, Y.M., Chan, H.Y.E., Huang, Y. and Chen, Z.Y. (2007) Green Tea Catechins Upregulate Superoxide Dismutase and Catalase in Fruit Flies. Molecular Nutrition \& Food Research, 51, 546-554. http://dx.doi.org/10.1002/mnfr.200600238

[22] Peng, C., Chan, H.Y., Li, Y.M., Huang, Y. and Chen, Z.Y. (2009) Black Tea Theaflavins Extend the Lifespan of Fruit Flies. Experimental Gerontology, 44, 773-783. http://dx.doi.org/10.1016/j.exger.2009.09.004 